\title{
Nasolacrimal system aeration on computed tomographic imaging: effects of patient positioning and scan orientation
}

This article was published in the following Dove Press journal:

Clinical Ophthalmology

II March 2015

Number of times this article has been viewed

\section{Craig N Czyz' \\ Thomas S Bacon ${ }^{2}$ \\ Andrew W Stacey ${ }^{3}$ \\ Eva N Cahill ${ }^{4}$ \\ Bryan R Costin ${ }^{5}$ \\ Boris I Karanfilov 6 \\ Kenneth V Cahill ${ }^{5}$}

'Section Oculofacial Plastic and Reconstructive Surgery, Ohio

University/OhioHealth, ${ }^{2}$ Department

of Medical Education, Mount Carmel

Health Systems, Columbus, OH, USA;

${ }^{3}$ Department of Ophthalmology,

University of Michigan, Ann Arbor,

MI, USA; ${ }^{4}$ Department of Biology,

Wittenberg University, Springfield,

$\mathrm{OH}, \mathrm{USA} ;{ }^{5}$ Department of

Ophthalmology, William H Havener

Eye Institute, Ohio State University

Wexner Medical Center, Columbus,

$\mathrm{OH}, \mathrm{USA} ;{ }^{6}$ The Sinus Institute of

Ohio, Dublin, OH, USA
Correspondence: Craig N Czyz 262 Neil Ave, Suite 430, Columbus, $\mathrm{OH} 4320 \mathrm{I}$, USA

$\mathrm{Tel}+$ I 6I4 22I 7464

Email Dsp4000@aol.com
Purpose: To determine the impact of patient positioning and scan orientation on the appearance of air in the nasolacrimal drainage system on computed tomography (CT) imaging, and the repeatability of the observations.

Methods: This was a retrospective analysis of CT images for 92 patients.

Results: Air was found to be present more fully in the upright-position group as compared with the supine-position group. Comparing axial and coronal scan orientation, no difference in aeration was found, except for the nasolacrimal duct in the upright-position group.

Conclusion: Patient position should be accounted for in diagnostic conclusions and treatment decisions based on $\mathrm{CT}$.

Keywords: axial, coronal, nasolacrimal sac, nasolacrimal duct

\section{Introduction}

Computed tomographic (CT) imaging is commonly used in the evaluation of periocular pathology, secondary to its widespread availability, detailed imaging, and short scan time. In recent years, numerous studies have used CT as a primary tool in detailing nasolacrimal drainage system (NLDS) anatomy and how variations may relate to drainage dysfunction. ${ }^{1-8}$ The majority of these reports have focused on structural variations, such as nasolacrimal duct (NLD) diameter and area, nasolacrimal volume, or duct angle in relation to the nasal floor, with few studies detailing the presence or absence of air on nasolacrimal imaging. ${ }^{7,8}$

Current research shows it is not uncommon to find air in one or both of the NLDs. ${ }^{6,8}$ The significance of this finding is not well elucidated and is historically regarded as a normal variation among individuals. This lack of information regarding the presence of air, or lack thereof, has led to an attempt at correlating nasolacrimal aeration or opacity with proximal sinus disease. A report by Loftus et al revealed no statistically significant difference in NLD opacification and ipsilateral sinus disease. ${ }^{8}$ The lack of success in correlating NLD aeration with proximal craniofacial as well as intrinsic NLD pathology may be due in part to the lack of data surrounding factors influencing the presence of air in the NLDS on CT imaging.

The intention of this study is to further advance the understanding of nasolacrimal imaging by evaluating, not only the presence or absence of air but also, the location of air and how it relates to patient position and scan orientation. These findings may allow for future correlations to pathologic states and/or be used to guide treatment. 


\section{Materials and methods}

A retrospective analysis of maxillofacial and sinus CT images was conducted, after protocol approval by The Mount Carmel Institutional Review Board. Patients were randomly selected from a hospital system and sinus institute radiology databases, with scan dates from 2008 to 2011. Patients were selected for the study, with the following exclusion criteria: age less than 18 years, history of facial and/or orbital trauma, preexisting nasolacrimal disease and/or its associated symptoms (eg, epiphora), pathology distorting visualization of the NLDS, preexisting sinus disease, and prior sinus or nasolacrimal surgery.

A total of 52 patients underwent supine axial imaging using a GE Optima CT660 (GE Healthcare, Little Chalfont, UK) or a Toshiba Aquilion 320 (Toshiba Medical Systems Corp, Tokyo, Japan), with images obtained at $0.2 \mathrm{~mm}$ intervals. Coronal reconstructions were generated using built-in system software for the respective scanner. A total of 40 individuals were scanned upright using an Iluma Cone Beam CT scanner (Imtec Imaging, Oklahoma City, OK, USA). Images were obtained at $0.4 \mathrm{~mm}$ intervals in both axial and coronal planes.

Scans were independently reviewed by three blinded observers for the presence of air within the NLDS for the right and left sides. If air was present, its location was noted as being in the nasolacrimal sac (NLS) and/or NLD, and further classified as partially or fully aerated (Figures 1-5). "Fully aerated" was applied to describe a continuous column of air filling the entirety of the lumen/sac, thus a fully aerated system was defined as a continuous column of air filling the entirety of the NLD and NLS. The findings were recorded for both coronal and axial images on all 92 patients. This

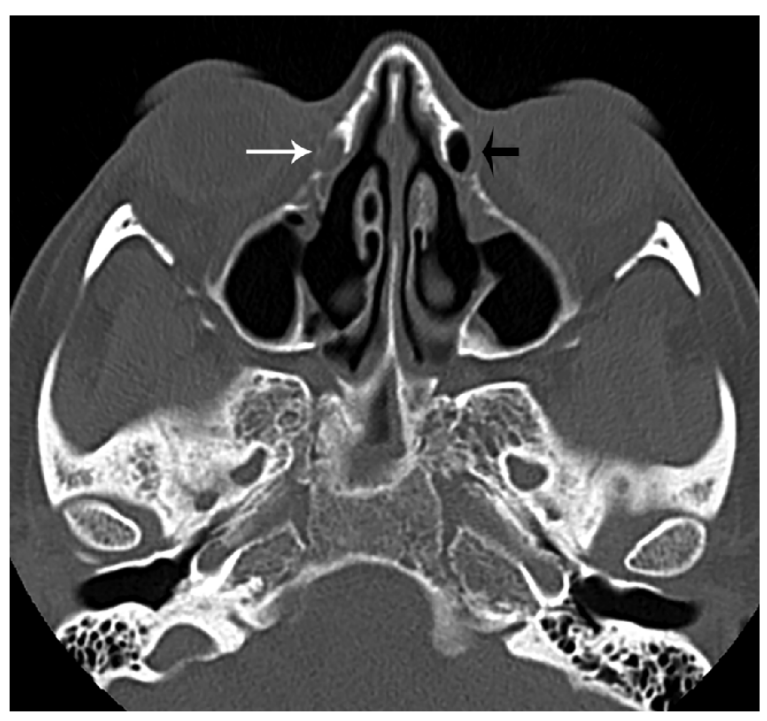

Figure I Axial image illustrating a fully opacified (white arrow) and a fully aerated (black arrow) lacrimal sac.

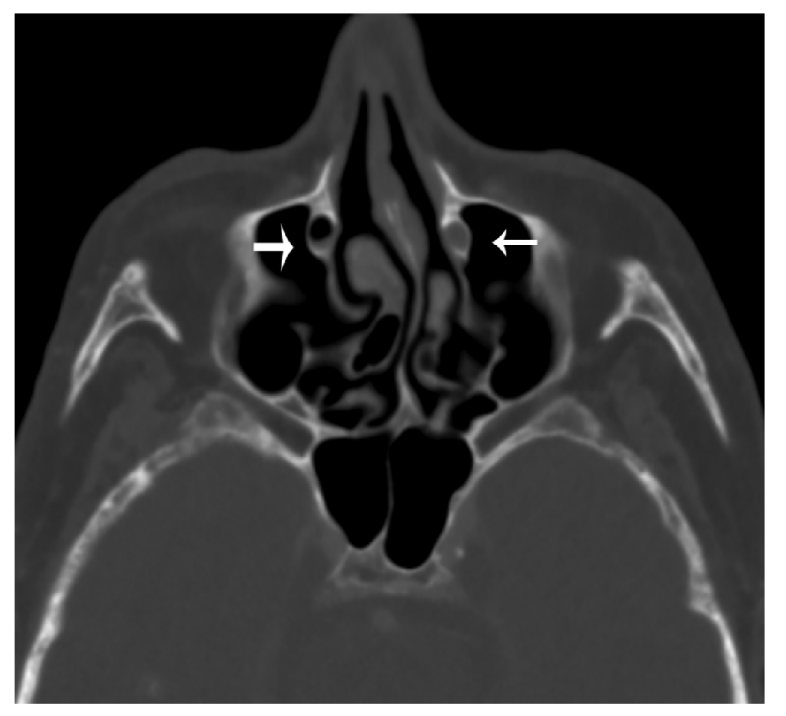

Figure 2 Axial image illustrating an opacified (small arrow) and a partially aerated (large arrow) nasal lacrimal duct.

resulted in four independent images per patient reviewed by three independent observers, for a cumulative 1,104 observations.

An a priori power analysis was not completed as there were no previously reported data available to estimate the differences in aeration between groups. Chi-square tests were used to test differences in observed frequencies of aeration between the groups. Four patients were scanned in both the upright and supine position. In this case, Fisher's exact test was used, due to the sparse numbers in each aeration category. Statistical significance was reported at the 0.05 alpha level, with two-tailed $P$-values. The R statistical package was used for data analysis. ${ }^{9}$ The use of multiple comparison correction was not indicated.

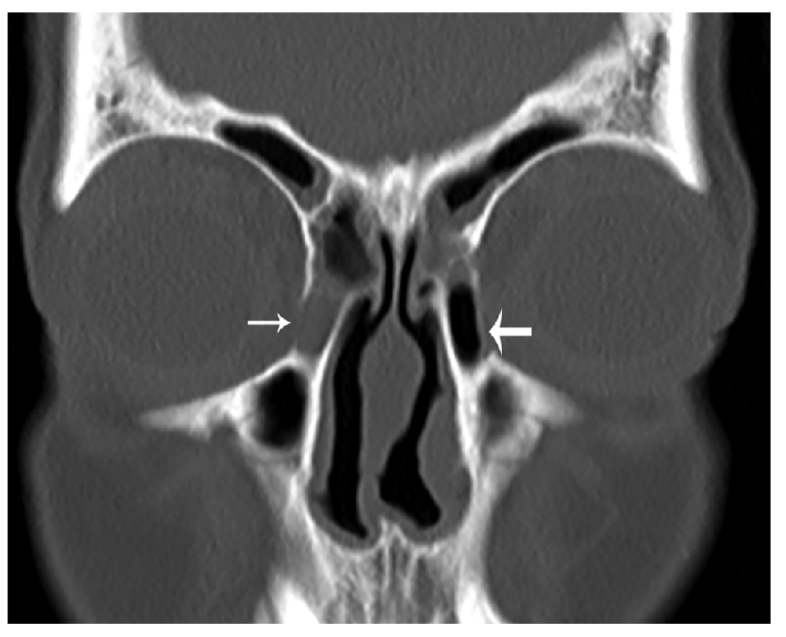

Figure 3 Coronal image illustrating an opacified (small arrow) and a fully aerated (large arrow) lacrimal sac. 


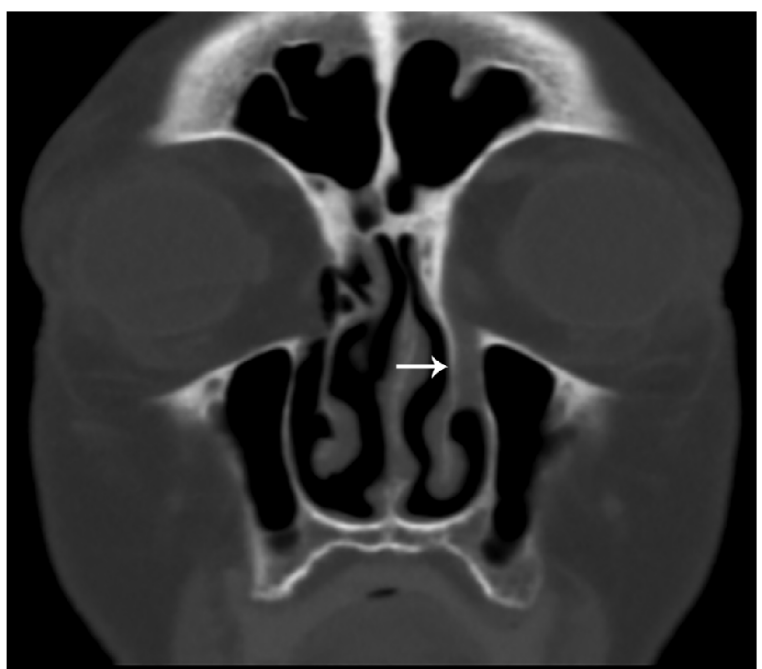

Figure 4 Coronal image illustrating an opacified nasolacrimal duct (arrow). Notes: Due to patient rotation, the contralateral duct cannot be viewed in this frame.

\section{Results}

A total of 184 NLDSs, from 92 patients (60 females and 32 males) with average age 48.5 years (range: $24-78$ years, standard deviation [SD]: 14.8 years), were included in the study. The reviewers showed excellent reliability, with all three individuals agreeing on aeration findings in $94.3 \%$ of NLS images, $93.5 \%$ of NLD images, and $90.5 \%$ of entire NLDSs.

Overall, air was identified in some portion of the NLDS in $30 \%$ of the scans, with air being fully visualized throughout the entire NLDS in $12 \%$ of scans (Table 1). Full aeration of the NLDS was significantly more common in

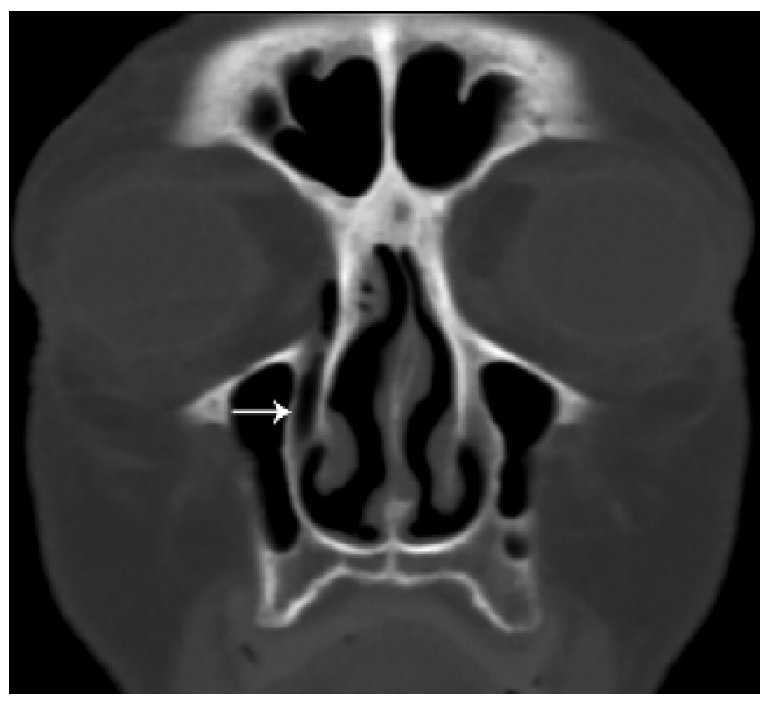

Figure $\mathbf{5}$ Coronal image illustrating a fully or partially aerated nasolacrimal duct (arrow).

Notes: The areas of density seen superiorly and inferiorly were interpreted as fluid within the duct by some reviewers.
Table I Overall identification of air in the nasolacrimal drainage system for all patient positions (supine/upright) and scan orientations (axial/coronal), for three reviewers

\begin{tabular}{llll}
\hline & No air & Partial air & Full air \\
\hline NLS & $72 \%$ & $12 \%$ & $16 \%$ \\
NLD & $76 \%$ & $11 \%$ & $14 \%$ \\
NLDS & $70 \%$ & $18 \%$ & $12 \%$ \\
\hline
\end{tabular}

Note: $(n=1,104)$.

Abbreviations: NLD, nasolacrimal duct; NLDS, nasolacrimal drainage system; NLS, nasolacrimal sac.

upright-position patient scans than in supine-position patient scans. Table 2 demonstrates that $21 \%$ of upright-position scans of the NLS resulted in full aeration, while only $12 \%$ of supine-position scans of the NLS were found to have full aeration ( $P=0.00007$, chi-square test). Similar results were found for the NLD, with $21 \%$ of upright-position scans resulting in full aeration, while only $8 \%$ of supine-position scans of the NLD resulted in full aeration $\left(P=5.0 \mathrm{e}^{-11}\right.$, chi-square test).

Additional analysis was performed on four patients who were scanned in both positions, upright and supine. These results showed a similar trend as those of the independently scanned groups. Complete aeration was seen more often in the upright position compared with the supine position in both the NLS ( $38 \%$ vs $35 \%$, respectively) and NLD ( $48 \%$ vs $35 \%$, respectively) (Table 3 ). The trend in this small sample was not statistically significant at the alpha $=0.05$ level and is only reported as anecdotal evidence (Fisher's exact test utilized).

On CT scan, aeration of the NLDS was further analyzed by comparing aeration results based on scan orientation (ie, axial vs coronal images). Table 4 presents the results of the data categorized first by scan position (upright vs supine) and further by scan orientation (axial vs coronal). The previous trend of more fully aerated NLS and NLD with upright patient positioning was again seen even when the data were controlled for the variable of scan orientation. The difference between aeration patterns of axial and coronal images was compared in four groups: supine NLS, upright NLS, supine NLD, and upright NLD. In all but one group, there was no statistical difference between the aeration results of axial and coronal scans. Upright images of the NLD did show a modest difference $(P=0.02$, chi-square test) between axial and coronal images. However, in these images, coronal views demonstrated both a higher percent of absent aeration and higher percent of full aeration. This result was due to the fact that fewer scans in this category resulted in partial aeration and, therefore, had minimal clinical significance. 
Table 2 Comparison in the aeration patterns for two scan positions (upright and supine), independent of scan orientation

\begin{tabular}{lllllll}
\hline & Position & N & No air & Partial air & Full air & P-value (chi-square test) \\
\hline NLS & Supine & 624 & $74 \%$ & $14 \%$ & $12 \%$ & 0.00007 \\
& Upright & 480 & $70 \%$ & $9 \%$ & $21 \%$ & \\
\multirow{2}{*}{ NLD } & Supine & 624 & $79 \%$ & $13 \%$ & $8 \%$ & $5.0 \mathrm{e}^{-11}$ \\
& Upright & 480 & $71 \%$ & $8 \%$ & $21 \%$ & \\
\hline
\end{tabular}

Notes: Both axial and coronal images are included.

Abbreviations: N, total number of observations; NLD, nasolacrimal duct; NLS, nasolacrimal sac.

\section{Discussion}

The presence of air within the NLDS on CT imaging is an infrequent finding, with images showing an absence of air $70 \%$ of the time. This supports previously published literature citing that approximately $30 \%$ of individuals scanned are found to have air within the NLDS, without regard to patient positioning or scan orientation. ${ }^{4}$ The data analysis from our sample revealed that patient position affects this CT finding. Patients scanned in the upright position were found to have air visualized more frequently and more fully than their supine counterparts, in both the NLS and NLD (Table 2). This finding is consistent with a trend observed in a subset of four patients who underwent both supine and upright imaging (Table 3).

Increased aeration on upright- versus supine-position scans supports the notion that gravity plays an important role in nasolacrimal drainage. While several theories exist concerning the exact muscular actions and resultant pressures involved in lacrimal drainage, the imaging data reinforces the role that patient position plays in lower nasolacrimal system mechanics. It is hypothesized that changes in aeration via position reflect gravitational forces; however, additional factors, such as lacrimal pump mechanisms and, to a lesser extent, pressure gradients, also contribute to decreased tear drainage. ${ }^{10-12}$ It also remains a possibility that supine positioning results in dependent congestion of the NLDS.

When analysis was conducted controlling for patient position, the results revealed that aeration of the NLDS can be determined equally as well on axial and coronal scans (Table 4). When factoring in patient position, upright scanning continued to produce more fully aerated NLDS components, regardless of scan orientation. The one group that did show a statistically significant difference (upright NLD) for axial vs coronal, did so as a result of the confounding variable effect of the "partial" aeration group. Ultimately, this result may be statistically significant, but it is not clinically relevant. In essence, there is no supporting evidence to suggest that scan orientation affects the ability to identify aeration, or lack thereof, in the NLDS in normal individuals.

The data suggest that patient position should be considered when interpreting imaging performed during the diagnostic evaluation of the NLDS. Accounting for factors altering image outcome, such as patient position, may allow both clinicians and researchers to more reliably correlate nasolacrimal system aeration findings with NLDS dysfunction as well as proximal pathology. Furthermore, CT is used in both diagnostic evaluation and preoperative planning for surgical intervention of lacrimal dysfunction. It has been shown that aerated NLSs can appear larger than normal on $\mathrm{CT}$, resulting in mistaken conclusions about their pathologic potential. This subsequently can erroneously influence the timing, approach, and decision to operate. ${ }^{4}$ Thus, awareness of the impact patient positioning has on the appearance of nasolacrimal structures can provide additional context in the interpretation of NLDS imaging on CT.

Limitations to this study include the subjective interpretation of radiographic imaging and selection bias. To minimize interpretation bias, the images were reviewed by three reviewers, all of whom were found to have excellent

Table 3 The effects of positioning in a subset of four individuals who underwent both supine and upright imaging

\begin{tabular}{|c|c|c|c|c|c|}
\hline & Position & No air & Partial air & Full air & $P$-value (Fisher's exact test) \\
\hline \multirow[t]{2}{*}{ NLS } & Supine & $63 \%$ & $2 \%$ & $35 \%$ & \multirow{2}{*}{0.15} \\
\hline & Upright & $50 \%$ & $12 \%$ & $38 \%$ & \\
\hline \multirow[t]{2}{*}{ NLD } & Supine & $60 \%$ & $5 \%$ & $35 \%$ & \multirow{2}{*}{0.47} \\
\hline & Upright & $50 \%$ & $2 \%$ & $48 \%$ & \\
\hline
\end{tabular}

Notes: The aeration results of supine positioning on NLS are compared to the results of upright positioning on the NLS, using a chi-square analysis. The same comparison is made between aeration of the NLD in supine and upright positions $(n=48)$.

Abbreviations: NLD, nasolacrimal duct; NLS, nasolacrimal sac. 
Table 4 Nasolacrimal system aeration patterns when images are categorized by scan position (supine vs upright), location (NLS vs NLD), and scan orientation (coronal vs axial)

\begin{tabular}{|c|c|c|c|c|c|c|c|}
\hline & & & $\mathbf{N}$ & No air & Partial air & Full air & $P$-value (chi-square test) \\
\hline \multirow[t]{4}{*}{ Supine } & \multirow[t]{2}{*}{ NLS } & Coronal & 312 & $74 \%$ & $13 \%$ & $13 \%$ & \multirow[b]{2}{*}{0.62} \\
\hline & & Axial & 312 & $74 \%$ & $15 \%$ & $11 \%$ & \\
\hline & \multirow[t]{2}{*}{ NLD } & Coronal & 312 & $79 \%$ & $15 \%$ & $6 \%$ & \multirow{2}{*}{0.14} \\
\hline & & Axial & 312 & $80 \%$ & $11 \%$ & $9 \%$ & \\
\hline \multirow[t]{4}{*}{ Upright } & \multirow[t]{2}{*}{ NLS } & Coronal & 240 & $70 \%$ & $9 \%$ & $21 \%$ & \multirow{2}{*}{0.94} \\
\hline & & Axial & 240 & $70 \%$ & $10 \%$ & $20 \%$ & \\
\hline & \multirow[t]{2}{*}{ NLD } & Coronal & 240 & $73 \%$ & $4 \%$ & $23 \%$ & \multirow{2}{*}{0.02} \\
\hline & & Axial & 240 & $69 \%$ & $11 \%$ & $20 \%$ & \\
\hline
\end{tabular}

Notes: The aeration results of coronal and axial scans are compared for four groups: supine NLS, upright NLS, supine NLD, and upright NLD. Abbreviations: $\mathrm{N}$, number of observations; NLD, nasolacrimal duct; NLS, nasolacrimal sac.

consistency, with agreement on greater than $90 \%$ of images. Selection bias is possible, as upright images were obtained from a sinus institute. However, as detailed in the "Materials and methods section", patients with preexisting sinus pathology were excluded from the study. An additional source of error was the use of three different CT scanners to obtain imaging; however all images were high definition, based on cut size, and viewed on the same reviewing software.

Air in the NLDS has been described as an uncommon finding on CT imaging. However, where, when, or why it appears has not been well-described. By reviewing variables influencing imaging interpretation, this study adds to the body of literature regarding CT diagnosis of NLDS dysfunction. The findings may also aid in diagnostic evaluation and preoperative planning, by identifying variables affecting CT imaging results.

\section{Disclosure}

The authors report no conflicts of interest in this work.

\section{References}

1. McCormick A, Sloan B. The diameter of the nasolacrimal canal measured by computed tomography: gender and racial differences. Clin Experiment Ophthalmol. 2009;37(4):357-361.

2. Janssen AG, Mansour K, Bos JJ, Castelijns JA. Diameter of the bony lacrimal canal: normal values and values related to nasolacrimal duct obstruction: assessment with CT. AJNR Am J Neuroradiol. 2001;22(5): 845-850.

Clinical Ophthalmology

\section{Publish your work in this journal}

Clinical Ophthalmology is an international, peer-reviewed journal covering all subspecialties within ophthalmology. Key topics include: Optometry; Visual science; Pharmacology and drug therapy in eye diseases; Basic Sciences; Primary and Secondary eye care; Patient Safety and Quality of Care Improvements. This journal is indexed on

Submit your manuscript here: http://www.dovepress.com/clinical-ophthalmology-journal
3. Ramey NA, Hoang JK, Richard MJ. Multidetector CT of nasolacrimal canal morphology: normal variation by age, gender, and race. Ophthal Plast Reconstr Surg. 2013;29(6):475-480.

4. Groell R, Schaffler GJ, Uggowitzer M, Szolar DH, Muellner K. CTanatomy of the nasolacrimal sac and duct. Surg Radiol Anat. 1997; 19(3):189-191.

5. Lee H, Ha S, Lee Y, Park M, Baek S. Anatomical and morphometric study of the bony nasolacrimal canal using computed tomography. Ophthalmologica. 2012;227(3):153-159.

6. Russell EJ, Czervionke L, Huckman M, Daniels D, McLachlan D. CT of the inferomedial orbit and the lacrimal drainage apparatus: normal and pathologic anatomy. AJR Am J Roentgenol. 1985;145(6):1147-1154.

7. Lefebvre DR, Freitag SK. Update on imaging of the lacrimal drainage system. Semin Ophthalmol. 2012;27(5-6):175-186.

8. Loftus WK, Kew J, Metreweli C. Nasolacrimal duct opacity on CT. Br J Radiol. 1996;69(823):630-631.

9. R Core Team. R: A Language and Environment for Statistical Computing. Vienna: R Foundation for Statistical Computing, 2014.

10. Hill JC, Apt R, Smirmaul HJ. Lacrimal pump pressure patterns. Can J Ophthalmol. 1975;10(1):25-31.

11. Lee MJ, Kyung HS, Han MH, Choung HK, Kim NJ, Khwarg S. Evaluation of lacrimal tear drainage mechanism using dynamic fluoroscopic dacryocystography. Ophthal Plast Reconstr Surg. 2011;27(3): 164-167.

12. Groessl SA, Sires BS, Lemke BN. An anatomical basis for primary acquired nasolacrimal duct obstruction. Arch Ophthalmol. 1997;115(1): $71-74$.

PubMed Central and CAS, and is the official journal of The Society of Clinical Ophthalmology (SCO). The manuscript management system is completely online and includes a very quick and fair peer-review system, which is all easy to use. Visit http://www.dovepress.com/ testimonials.php to read real quotes from published authors. 